approved for the treatment of active SLE patients not responding to standard of care, without active kidney or neuropsychiatric (NP)involvement.

Objectives: Aim of the study was to analyse 36 months survival of BLM treatment, causes of withdrawal in a monocentric cohort of SLE patients followed-up in a daily practice setting.

Methods: The study was proposed to all the patients strarting BLM. After the informed consent was obtained, demographic, clinical and serological data, indication to BLM and concomitant therapies were registered. At baseline and at $6,12,24,36$ months of follow-up, disease activity (SLEDAI 2K), DAS28, C3 and C4 levels, anti-dsDNA status and weekly dose of glucocorticoids were recorded. Data were expressed as median-interquartile range; after 6,12,24,36 months, differences in all parameters compared to baseline were evaluated (Student $t$ test) The treatment survival was evaluated by Kaplan-Meier analysis. $P$ value $<0.05$ were considered significant

Results: We enrolled 39 Caucasian individual, 38 females, 1 male, with median age of 43 (IQR 7.5) years and median disease duration 14.5 (5.5) years. Indications for starting BLM were: mucocutaneous involvement $(n=11,28 \%)$, arthritis $(n=25,64 \%)$, systemic symptoms $(n=3,7 \%)$ and lung involvement (1 pt,2\%). At baseline, all the patients were taking PDN;97\% hydroxychloroquine,23\% mycophenolate mofetil,23\% azathioprine, $5 \%$ cyclosporine, 7\% methotrexate and $2 \%$ thalidomide. Table 1 summarises trend of SLEDAI 2K, C3 and C4, DAS28 (for articular involvement), prednisone dose and percentage of patients positive for anti-dsDNA during the follow-up. Fourteen out of the 39 patients $(35.8 \%)$ reached 12 months of observation and only 4 the 24 months, 3 the 36 months. Figure 1 shows the survival curve of Belimumab. In 8 patients (20\%) adverse events were the cause of BLM withdrawal (severe infection in one patient, severe bradychardia in one and acute infusion reaction in another one). In 4 patients (10\%) BLM was discontinued for lack of efficacy(articular and skin manifestations)after 4, 8, 12 and 23 months respectively; in 6 patients (15\%) for loss of efficacy; one patient developed a severe NP flare after 2nd BLM infusion and was admitted in our hospital for depression. One patient was lost during the follow-up. Two patients withdrew BLM therapy to plan a pregnancy. We found significant reduction of PDN week levels, and improvement of C4 and aDNA levels at T6; as for disease activity, we found significant reduction of DAS28 at T6, and of SLEDAI-2k at T6 and T12.

\begin{tabular}{lccc}
\hline & T0 & T6 & T12 \\
\hline $\mathrm{N}^{\circ}$ of patients & 39 & 25 & 14 \\
PDN mg/sett & $52.5(39.5)$ & $35(17.5)$ & $35(0)$ \\
SLEDAl 2K & $6(3.5)$ & $2(2)$ & $2(0.75)$ \\
C3 & $79(19.75)$ & $84.5(17.5)$ & $73.5(9.75)$ \\
$\mathrm{C} 4$ & $13.5(12.75)$ & $7(10) \S$ & $10(10.25)$ \\
$\mathrm{aDNA}(\%)$ & 100 & 20 & 5 \\
DAS28 & $4.32(0.7)$ & $3.3(1.33)$ & $3(0.65)$ \\
& & &
\end{tabular}

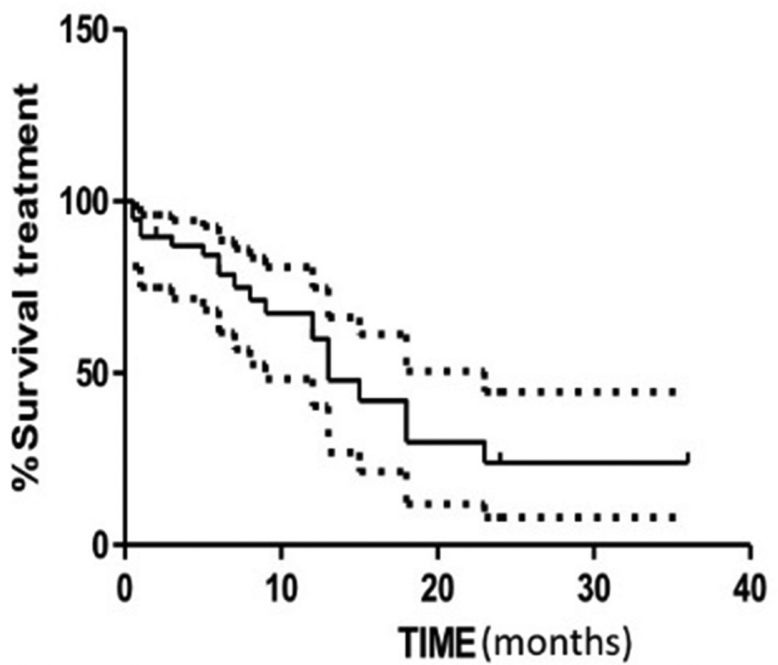

Abstract FRI0321 - Figure 1. the figure shows patients mantanining treatment with Belimumab

Conclusions: In our monocentric cohort of SLE patients, BLM demonstrated to be effective on disease activity and serology and led to a significant decrease of glucocorticoids dose; the main causes of BLM withdrawal were adverse events or disease flares.
Disclosure of Interest: None declared

DOI: 10.1136/annrheumdis-2018-eular.6313

\section{FRI0322 \\ RATIONALE FOR THE ATACICEPT DOSE FOR A PHASE III STUDY IN PATIENTS WITH HIGHLY ACTIVE AND AUTO-ANTIBODY POSITIVE SLE}

J. Shen $^{1}$, O. Papasouliotis ${ }^{2}$, E. Samy ${ }^{1}$, P. Haselmayer ${ }^{3}$, P. Chang ${ }^{1}$, V. Ona ${ }^{1}$, A. Kao ${ }^{1}{ }^{1}$ EMD Serono Research and Development Institute, Inc. (a business of Merck KGaA, Darmstadt, Germany), Billerica, USA; ${ }^{2}$ Merck Institute for Pharmacometrics (an affiliate of Merck KGaA, Darmstadt, Germany), Lausanne, Switzerland; ${ }^{3}$ Merck KGaA, Darmstadt, Germany

Background: Atacicept targets the B-cell stimulating factors BLyS and APRIL, and is currently in clinical development for the treatment of patients (pts) with active, auto-antibody positive SLE.

Objectives: Here, we evaluated integrated nonclinical, clinical and exposureresponse $(E-R)$ data from atacicept studies to determine an appropriate atacicept dose for a Phase (P) III study in SLE pts with high disease activity (HDA).

Methods: Nonclinical efficacy and pharmacokinetic (PK)/pharmacodynamic data for atacicept were obtained from two murine models: An F1 hybrid NZBWF1/J spontaneous SLE model (given mouse Fc-protein control or mouse TACl-Fc $5 \mathrm{mg} / \mathrm{kg}$ intraperitoneal [IP] three times per week) and a 4-Hydroxy-3-nitrophenylacetyl-Keyhole Limpet Haemocyanin (NP-KLH) vaccinated C57BL/6 model to assess immunomodulation (given control protein $10 \mathrm{mg} / \mathrm{kg}$ or atacicept 1,3 or $10 \mathrm{mg} / \mathrm{kg}$ IP every third day). Clinical PK, efficacy, safety and E-R data were obtained from a PI PK study in healthy participants (Study 022; single dose atacicept: 25,75 or $150 \mathrm{mg}$ ) and two PII studies in pts with autoantibody-positive SLE (APRIL-SLE [EudraCT 2007-003698-13] and ADDRESS II [EudraCT 2013002773-21]; randomization [1:1:1] to once weekly [QW] subcutaneous [SC] injections of atacicept [75 or $150 \mathrm{mg}$ ] or placebo [PBO]). In APRIL-SLE, the primary endpoint was the proportion of pts with BILAG A/B flare over 52 weeks. In ADDRESS II, the primary endpoint was SRI-4 response at Week 24. SRI-6 response was analysed post-hoc in pts with HDA (SLEDAI-2K $\geq 10$ ) at Screening. A population PK model was established using data from the PI and PII studies. Population PK model-derived exposure vs probability of clinical response (BILAG A/B flare, SRI-4, SRI-6) was assessed, and an exploratory analysis of exposure vs safety performed.

Results: TACl-Fc $5 \mathrm{mg} / \mathrm{kg}$ prevented proteinuria development and glomerular damage in the $\mathrm{F} 1$ hybrid NZBWF1/J spontaneous SLE model. Anti-KLH IgG decreased markedly in atacicept-treated NP-KLH vaccinated mice ( $>50 \%$ reduction vs control protein at all doses), with mean atacicept serum trough concentrations $\left(C_{\min }\right)$ of $\sim 2.3 \mu \mathrm{g} / \mathrm{mL}(1 \mathrm{mg} / \mathrm{kg}) \sim 5 \mu \mathrm{g} / \mathrm{mL}(3 \mathrm{mg} / \mathrm{kg})$ and $\sim 8.5 \mu \mathrm{g} / \mathrm{mL}$ $(10 \mathrm{mg} / \mathrm{kg})$. In post-hoc analyses of the proportion of pts with BILAG A/B flare and time to flare in APRIL-SLE, and SRI-4 and SRI-6 response in SLE pts with HDA in ADDRESS II, treatment with atacicept $150 \mathrm{mg} \mathrm{QW}$ demonstrated greater clinical response vs PBO. In both studies, atacicept E-R relationships based on population PK-derived exposure were observed. For maximal flare reduction, an atacicept exposure of AUC $\geq 1 \mathrm{mg}$ *hr/mL was identified. This exposure was more achievable with 150 than $75 \mathrm{mg} \mathrm{(60 \%} \mathrm{vs} 15 \%$ probability) and corresponded to an atacicept $C_{\min }$ of $5 \mu \mathrm{g} / \mathrm{mL}$, which was similar to the $C_{\min }$ values observed in the NP-KLH vaccinated mouse model. Both atacicept 150 and $75 \mathrm{mg}$ had acceptable safety profiles in SLE pts, with no apparent E-R relationship observed for serious infections.

Conclusions: Integrated nonclinical, clinical and E-R data demonstrate an acceptable benefit-risk profile for atacicept in SLE pts with HDA and support the selection of the $150 \mathrm{mg} \mathrm{QW}$ dose for a PIII study.

Disclosure of Interest: J. Shen Employee of: EMD Serono Research and Development Institute, Inc. (a business of Merck KGaA, Darmstadt, Germany), O. Papasouliotis Employee of: Merck Institute for Pharmacometrics, Lausanne, Switzerland (an affiliate of Merck KGaA, Darmstadt, Germany), E. Samy Employee of: EMD Serono Research and Development Institute, Inc. (a business of Merck KGaA, Darmstadt, Germany), P. Haselmayer Employee of: Merck KGaA, P Chang Employee of: EMD Serono Research and Development Institute, Inc. (a business of Merck KGaA, Darmstadt, Germany), V. Ona Employee of: EMD Serono Research and Development Institute, Inc. (a business of Merck KGaA, Darmstadt, Germany), A. Kao Employee of: EMD Serono Research and Development Institute, Inc. (a business of Merck KGaA, Darmstadt, Germany) DOI: 10.1136/annrheumdis-2018-eular.2050 